\title{
Role of Laser Induced Plasma in Propulsion
}

\author{
Athrva Pandhare, Abhishek Puri \\ Department of Aerospace Engineering, SRM University, Chennai, India \\ abhishekpuri_sanjeev@srmuniv.edu.in; athrva_atul@srmuniv.edu.in
}

\begin{abstract}
In this paper an effort is made to apply the principles of laser sustained plasma in jet propulsion. We have also tried to explore the potential of their use in the replacement of conventional jet propulsion systems and have tried to use the principles of laser induced plasma to produce viable thrust by irradiating the compressed air molecules by a high intensity pulsed laser. The temperature of the irradiated molecules of air increases, and consequently the air tends to undergo expansion. This expansion of air is done at the nozzles to produce viable thrust to drive the engine. One of the advantage of this propulsion system is that it doesn't require any significant changes in the design that is used in petroleum based jet propulsion systems.Furthermore, a theoretical analysis and comparison of the proposed propulsion system and the conventional systems is done in this paper and results obtained in the calculations are included. We have also included a design of the setup to be used in order to conduct a more precise and scientific experiment to study to performance of the proposed technique.
\end{abstract}

\section{Introduction}

Plasma (from Ancient Greek, meaning "mouldable substance") is a state of matter in which an ionised gaseous substance becomes highly electrically conductive to the point that long-range electric and magnetic fields dominate the behaviour of the matter. It is one of the four fundamental states of matter.

A plasma is referred to as being "hot" if it is nearly fully ionised, or "cold" if only a small fraction (for example 1\%) of the gas molecules are ionised, but other definitions of the terms "hot plasma" and "cold plasma" are common. Plasmas utilized in "plasma technology" ("technological plasmas") are usually cold plasmas in the sense that only a small fraction of the gas molecules are ionised. Plasma created by the radiation from focused laser beams was first observed with the advent of "large pulsed" Q-switched ruby laser.The plasmas that are formed by the spontaneous breakdown of the gas at the focus of the lens are sustained only for the duration of the incident laser pulses. However with the advent of continuous, high power carbon dioxide lasers, it became possible to sustain a plasma in a steady state condition near the focus of a laser beam.

The laser sustained plasma shares many characteristics with other gas discharges, however it is sustained through absorption of power from an optical beam by the inverse bremsstrahlung process. Since the optical frequency of the sustaining beam is greater than the plasma frequency the beam is capable of propagating well into the interior of the plasma where it is absorbed at high frequency near the focus. This is in contrast to the plasma sustained by high frequency electrical fields (microwave and electrode less discharge) that operate at frequencies below the plasma frequency and sustain the plasma through absorption within a thin layer near the plasma surface. This fundamental difference in the power absorption mechanism makes it possible to generate steady state plasma having maximum temperature of $10000 \mathrm{~K}$ or more in a small volume near the focus of the lens, far away from any confining structure.

This ability to sustain a plasma within a small, isolated volume at relatively high pressure and temperature has suggested a number of potential applications for the laser sustained plasma.

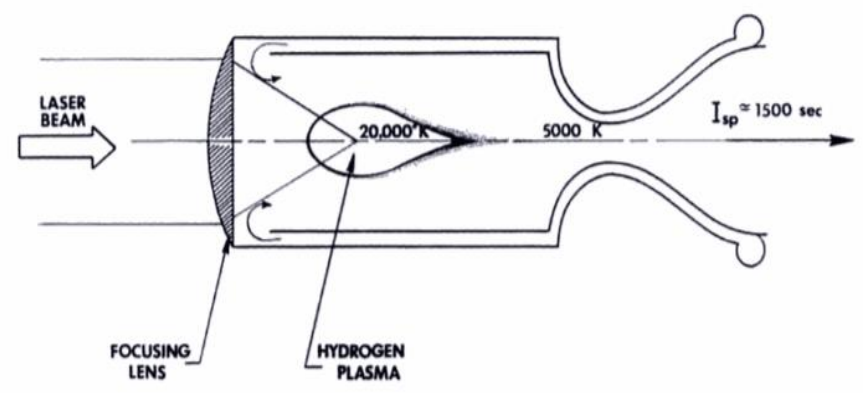

Fig. 1: Similar model but using hydrogen as a fuel. 
The frequency of optical fields, ( $28 \mathrm{THz}$ for the 10.6 micrometer carbon dioxide laser) used for the LSP is greater than the plasma frequency.[1]

\section{Literature Review}

\subsection{Laser Propulsion: Early Historical Background}

The idea to send a beam of light to a distant location and use its energy or its momentum has been around since antiquity, long before the laser was invented. Archimedes's mirrors reflecting sunlight and focusing it onto the Roman fleet of Commander Marcellus off the coast of Syracuse in 214 BC is the best-known example. True story or mere myth, the interesting point is that this story has persisted over more than 2000 years, demonstrating the attractiveness of such a capability. The first more-seriously-documented approaches to the application of directed light beams are found in the publications of the astronautic visionaries of the 20th century. In the years 1923 and 1924, the Russian pioneers Fridrikh Tsander and Konstantin Tsiolkovsky and, independently, the German Hermann Oberth mentioned the idea of propulsion by light pressure, leading to the concept of light, or solar, sail. However, the Russian work was virtually unknown to the West until the 1930s, when rocket technology had developed through the independent efforts of Oberth in Germany and Goddard in the United States.[2]

\subsection{Characteristics of the GE J79 Engine}

The General Electric J79 is an axial-flow turbojet engine built for use in a variety of fighter and bomber aircraft and a supersonic cruise missile, the J79 was produced by General Electric Aircraft Engines in the United States and under licence by several other companies. First flight using this engine was in 1955, 20 may.[3]

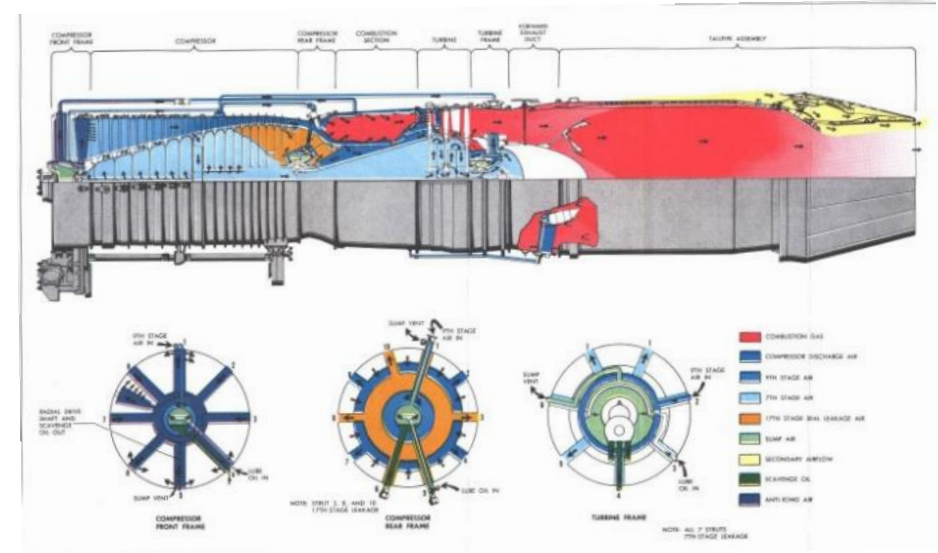

Fig. 2: The schematic diagram of the General Electric J79 showing the arrangement of internal parts [4].

Some of the aircraft in which this powerplant is used are Conveyer B-58 Hustler, Lockheed F-104 Star fighter, North American A-5 Vigilante. In 1959, the gas generator of the J79 was developed as a stationary 10MW-class(13000bhp) freeturbine turboshaft engine for naval power generation, and industrial use called LM1500. Its first application was in the research of hydrofoil USS plainview.

\subsection{The Laser-Induced Breakdown Process}

From electrical engineering, it is known that for sufficiently high electric field strengths in an insulating medium (e.g. air or glass) a breakdown can occur. This means that there is a kind of spark, and the medium becomes electrically conducting. The mechanism behind this effect is based on the acceleration of free electrons to high energies so that collisions with other atoms or molecules can lead to secondary free carriers. This starts an avalanche process, during which appreciable densities of free carriers can be built up within a short time. A plasma is formed, which can have a significant electrical conductivity. The plasma can be maintained by further current flow, which generates additional free carriers. [5]

Breakdown in air and in other transparent media can also be initiated by intense light, i.e. by electromagnetic waves with frequencies of hundreds of terahertz. The high optical intensities required can be reached in pulses as generated e.g. in a Q-switched laser (with nanosecond durations) or in a mode-locked laser and amplified in a regenerative 
amplifier (for pulse durations of picoseconds or femtoseconds). The intensity required for optical breakdown depends on the pulse duration. For example, for 1-ps pulses an optical intensity of $\approx 2 \times 10^{13} \mathrm{~W} / \mathrm{cm}^{2}$ is required for breakdown in air at normal pressure.

Interestingly, laser induced breakdown is possible at lower intensity levels if the air pressure is increased.

\subsection{Physical Mechanisms}

The detailed mechanisms behind breakdown with optical pulses are different from those for static electric fields, and also depend on the pulse duration. Particularly for femtosecond pulses, multiphoton ionization can efficiently generate free carriers in the initial phase of the pulse, followed by strong absorption by the generated plasma, which leads to further heating and ionization. For long pulses, multiphoton ionization is less important, and the breakdown starts primarily from the few carriers which are already present before the pulse. The random occurrence of such carriers makes optical breakdown less deterministic in the regime of longer pulses, whereas the breakdown threshold can be very well defined for femtosecond pulses.

\section{Working Principle}

The incoming laser beam is focussed into the combustion chamber where it sustains a plasma at temperature approximately 16000 to $20000 \mathrm{~K}$ in contrast to a normal combustion chamber the high temperature plasma occupies only a small fraction of the chamber volume and comes in contact with the incoming compressed air( having compression ratio from 50:1 to 130:1) to produce a flow having bulk temperature approximately $1198 \mathrm{~K}$ for an ambient air temperature of $300 \mathrm{~K}$ that can be expanded through nozzle to produce thrust. A detailed overview of the working of the proposed engine is as follows. The air is admitted into the engine through the inlet as is the case in any ordinary turbojet or turbofan engine. After this the air is compressed until the compression ratio reaches about 50:1 to 130:1 depending upon the altitude at which the aircraft is flying.

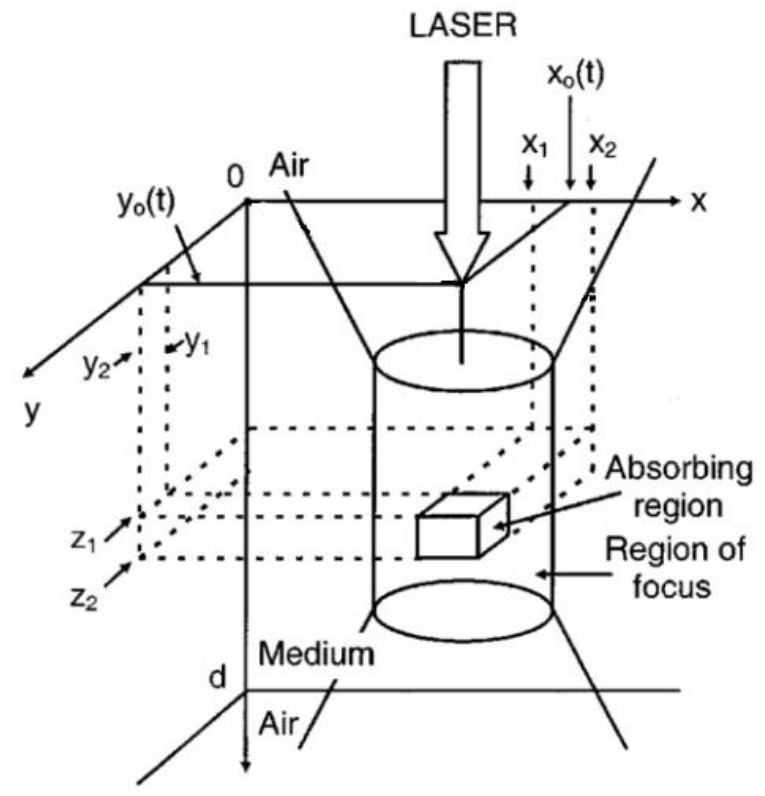

Fig. 3: Laser focusing in a region in space[6].

The above two steps are common to any ordinary engine but in the LSP based engine, the compressed air flows into a chamber in which a laser is housed, the focal point of the laser is along the axis of the engine and is well behind the turbine. 


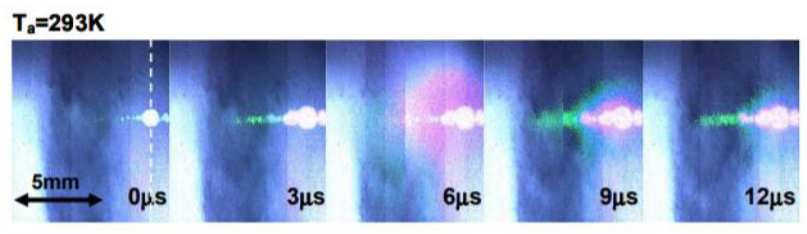

$T_{a}=390 K$

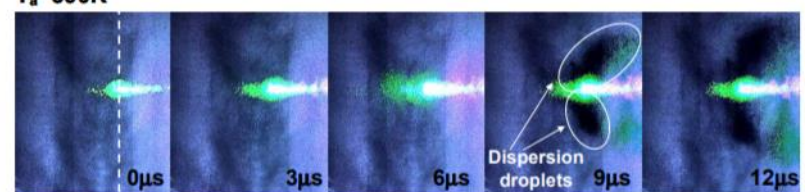

Fig. 3: The above diagram shows a time evolution of laser induced plasma in fuel spray. We are not using any fuel in our model, but the diagram is similar and can provide some insight [7].

The laser then produces a sustained plasma at its focus, the incoming air which comes in contact with the plasma is the heated and the bulk temperature is raised significantly, to calculate the bulk temperature, in addition to using principles of forced convection, (based on the fact that the plasma has a higher density) the steady flow energy equation can also be used. The heated and the intermediately pressurised air then passes through the turbine transferring some of its kinetic energy to the turbine, remaining air flows to the nozzle and by expanding at the nozzle produces the necessary thrust to propel the aircraft. However, due to the high temperature of the LSP a substantial portion of the absorbed power will be radiated from the plasma and absorbed by the chamber walls, consequently some of the energy radiated by the plasma is wasted, hence it needs to be ensures that the material used in the body of the engine has low thermal conductivity and a high melting point.It needs to be ensured that sufficient mixing of the inlet air and the high temperature plasma region near and at the LSP takes place to produce bulk temperature required for expansion at the nozzle inlet as well as for running the turbine.

It is quite conspicuous that the success of this model is dependent on two factors.

1. Being able to sustain plasma in a forced convective flow.

2. Being able to produce a uniform temperature distribution at the turbine.

Furthermore, the properties of nitrogen/ air plasma need to be studied [8].

\section{Mathematical Analysis}

For the mathematical analysis it is assumed that if the proposed engine can heat the incoming air to the temperatures that are reached in the conventional engines at the nozzles then the rate of expansion of the air at the nozzle of the proposed engine will be equal to the rate of expansion of the air at the nozzles of conventional engines, then assuming that the flow rate is the same for the proposed engine and the conventional jets the thrust produced will also be the same. Note that the geometry of the nozzle of both the engines is assumed to be identical. For the success of the proposed model it is important that the calculated bulk temperature is sufficiently high so as to accelerate the air to optimum velocities that are needed to produce thrust. The mathematical analysis for the same involves a control volume analysis of the combustion chamber to calculate the temperature at every section of the control volume in addition to calculating the bulk temperature.

Consider a control volume as shown in the figure which in our case is the combustion chamber. Let the flow of the air considered be a steady flow. Note that this mathematical analysis only provides general equations the can be used to calculate temperatures and pressures at different sections of the control volume.Applying the Energy Equation for steady flow, and Assuming that the work done is zero, furthermore assuming that the mass flow rate remains the same, [9]

We get that,

$$
\begin{aligned}
& \frac{\partial m}{\partial t}\left[u_{1}+p_{1} v_{1}+\frac{v_{1}^{2}}{2}+g z_{1}\right]+\frac{\partial Q}{\partial t}=\frac{\partial m}{\partial t}\left[u_{2}+p_{2} v_{2}+\frac{v_{2}^{2}}{2}+g z_{2}\right] \\
& \frac{\partial Q}{\partial t}=\frac{\partial m}{\partial t}\left[\left(u_{2}-u_{1}\right)+\left(p_{2} v_{2}-p_{1} v_{1}\right)+\left(\frac{v_{2}^{2}}{2}-\frac{v_{1}^{2}}{2}\right)+g\left(z_{2}-z_{1}\right)\right]
\end{aligned}
$$


Consider that $z_{2}-z_{1}=0$ then,

$$
\left.\frac{\partial Q}{\partial t}=\frac{\partial m}{\partial t}\left[\Delta h+\left({\frac{v_{2}}{2}}^{2}-{\frac{v_{1}}{2}}^{2}\right)\right)\right]
$$

If Q represents the total heat produced, then,

$$
Q=Q_{\text {air }}+Q_{\text {walls }}
$$

Where $Q_{\text {air }}$ represents the fraction of the heat from the plasma that is used to heat the air and $Q_{\text {walls }}$ represents the heat that is absorbed by the walls of the chamber,

And,

$$
\alpha=\frac{Q_{\text {air }}}{Q}
$$

$\alpha$ represents the fraction of the heat that is used in raising the temperature of the air and hence is equal to the ratio of the heat absorbed by the air to the total heat supplied by the plasma.

Then we get,

$$
\frac{\partial Q_{\text {air }}}{\partial t}=\alpha\left[\frac{\partial m}{\partial t}\left[\Delta h+\left(\left({\frac{v_{2}}{2}}^{2}-{\frac{v_{1}}{2}}^{2}\right)\right)\right]\right]
$$

Multiply both sides by $\frac{\partial t}{\partial m}$

$$
\dot{Q}_{\text {alr }}=\alpha\left[\left[\Delta h+\left(\left({\frac{v_{2}}{2}}^{2}-{\frac{v_{1}}{2}}^{2}\right)\right)\right]\right]
$$

But $Q_{\text {air }}$ can be written as,

$$
Q_{\text {air }}=(c \Delta T)_{\text {air }}
$$

Then for unit mass flow rate,

$$
(c \Delta T)_{a i r}=\alpha\left[\left[\Delta h+\left(\left(\frac{v_{2}^{2}}{2}-\frac{v_{1}^{2}}{2}\right)\right)\right]\right]
$$

This expression provides us a rough estimate to calculate the temperature at any considered section but to get a more precise value of the temperature a control volume analysis must be carried out.

Divide the control volume into infinite small cylindrical elements and assume that the pressure remains constant within each of these cylindrical elements. 


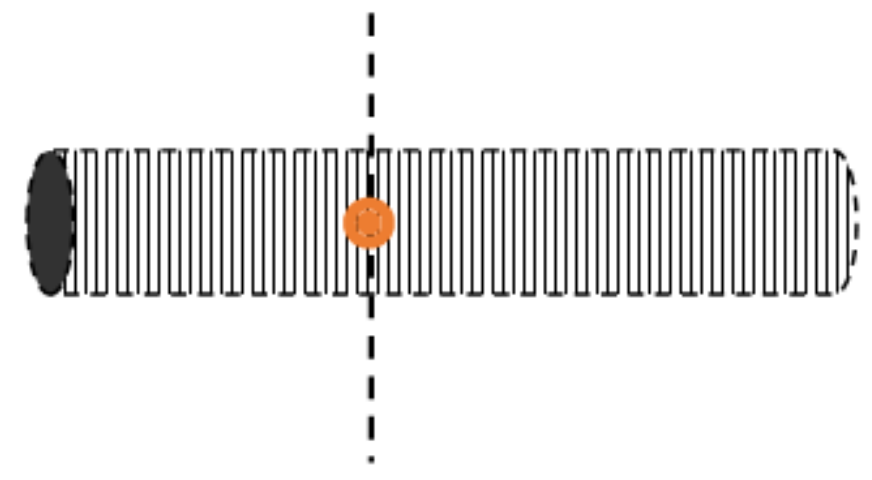

Fig. 4: Plasma generated at the axis of the cylinder[1].

It is assumed that before the air reaches the plasma all of the temperature change is brought about due to the compression from the compressors. Therefore in this analysis we only consider the elements after or downstream of the introduction of the plasma.

For strip 1

$$
\left[\Delta T c_{p}\right]_{1}=\alpha\left[\left[[\Delta h]_{1}+\left(\left({\frac{v_{2}}{2}}^{2}-{\frac{v_{1}}{2}}^{2}\right)\right)\right]\right]
$$

$T_{2}$ is the temperature of the air after it interacts with the plasma and $T_{1}$ is the temperature before interaction. Therefore $T_{1}$ is the temperature of the air that is achieved due to compression from the compressor. Hence during experimental analysis the value of $\mathrm{T}_{1}$ is known.

For strip 2

$$
\begin{gathered}
{\left[\Delta T c_{p}\right]_{2}=\alpha\left[\left[[\Delta h]_{2}+\left(\left({\frac{v_{3}}{2}}^{2}-\frac{v_{2}{ }^{2}}{2}\right)\right)\right]\right]} \\
\left.\left[\Delta T c_{p}\right]_{2}=\alpha\left[\left[\left[h_{3}-h_{2}\right]+\left({\frac{v_{3}}{2}}^{2}-\frac{v_{2}}{2}\right)\right)\right]\right]
\end{gathered}
$$

It is also considered that the enthalpy of the air within each individual strips also changes along with the velocity.

$$
\left[T_{3}-T_{2}\right]\left[c_{p}\right]_{2}=\alpha\left[\left[\left[h_{3}-h_{2}\right]+\left(\left({\frac{v_{3}}{2}}^{2}-{\frac{v_{2}}{2}}^{2}\right)\right)\right]\right]
$$

For strip 3

$$
\left[\Delta T c_{p}\right]_{3}=\alpha\left[\left[[\Delta h]_{3}+\left(\left({\frac{v_{4}}{2}}^{2}-{\frac{v_{3}}{2}}^{2}\right)\right)\right]\right]
$$




$$
\left[T_{4}-T_{3}\right]\left[c_{p}\right]_{3}=\alpha\left[\left[\left[h_{4}-h_{3}\right]+\left(\left({\frac{v_{4}}{2}}^{2}-{\frac{v_{3}}{2}}^{2}\right)\right)\right]\right]
$$

Repeating the same analysis for all the strips, We get a general formula,

$$
\left[T_{n+1}-T_{1}\right]\left[c_{p}\right]_{3}+\sum_{i=0}^{n} T_{n}\left[c_{p_{n-1}}-c_{p_{n}}\right]=\alpha\left[\left[\left[h_{n+1}-h_{1}\right]+\left(\left({\frac{v_{n+1}}{2}}^{2}-\frac{v_{1}{ }^{2}}{2}\right)\right)\right]\right]
$$

This equation helps in a more precise calculation of the temperature of any particular section of the control volume.

\section{Calculation for the Bulk Temperature}

It is known that for a static homogeneous fluid system,

$$
T_{b}=\frac{I}{C}
$$

Where $\mathrm{I}$ is the total enthalpy,

and $\mathrm{C}$ is the heat capacity.

However, the fluid system that we are dealing with is not a static homogeneous system hence this equation does not apply to the system as a whole however it does apply to each individual point of the system when considered separately.[10]

Assuming the pressure changes to be negligible in the local elements of fluid flow i.e. the pressure within each individual element of the control volume is constant.

Let the local velocity of the flow be $u$.

And the local temperature be $T$.

This temperature is a function of the special coordinates as there exists a temperature profile in the system.

The mass flow rate in through any section is,

$$
\dot{m}_{i n}=\rho u d A
$$

For two dimensional system,

$$
\dot{m}_{i n}=\rho u d x d y
$$

The enthalpy is,

$$
\rho u c_{p} T d A
$$

And the heat capacity is,

$$
\rho u c_{p} d A
$$

Then the bulk temperature is,

$$
T_{b}=\frac{\int \rho u c_{p} T d A}{\int \rho u c_{p} d A}
$$




$$
\begin{aligned}
& T_{b}=\frac{\int u T d A}{\int u d A} \\
& T_{b}=\frac{\int u T d A}{U A}
\end{aligned}
$$

This is the theoretical method to find the bulk temperature. However, this definition in Eq. (24) also makes it difficult to be measured in an experiment accurately. But an approximation can be done using the method suggested in the Fig. 4 below. Each white dot is the place where actual temperature and local velocity measurements are made while the flow persists. Such local values are approximated to represent the temperature for a control volume of the fluid region that surrounds those points (In Fig. 4, represented by the rectangles formed by the grid). An area weighted summation of all such temperature values should give an answer that is close to the RHS of Eq. (24). The fineness or coarseness of the grid in Fig. 4 determines the accuracy of the bulk temperature measurement.

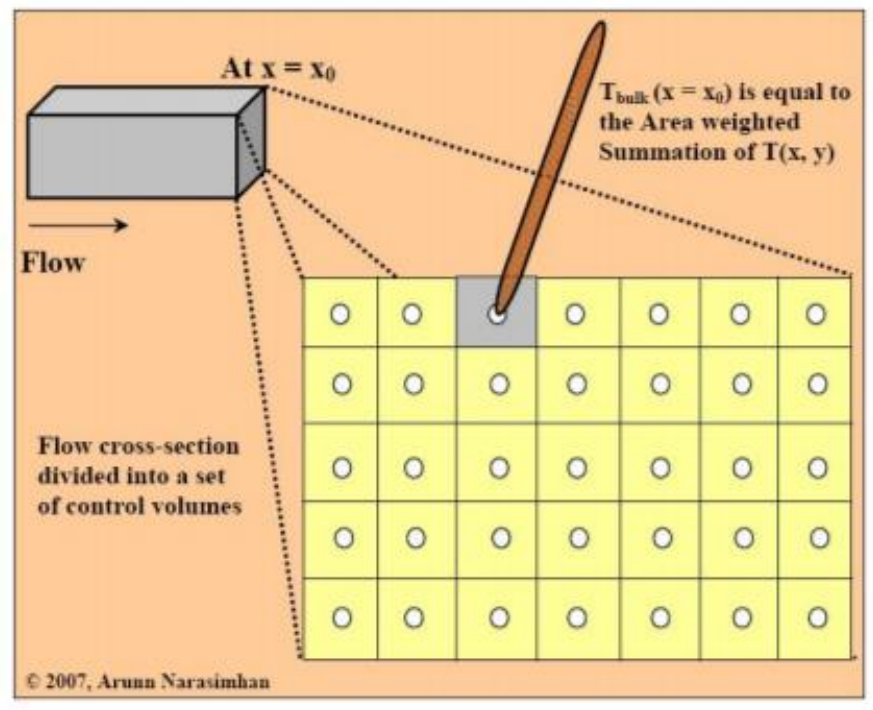

Fig. 5: Experimental determination of bulk temperature in a steady channel flow, forced convection situation taken [11].

The efficiency of the technique can be calculated as follows,

The input heat can be calculated from the rating of the laser used,

From eq. (5)

$$
\alpha=\frac{Q_{\text {air }}}{Q}
$$

Here $\alpha$ is the fraction of the incident heat that is actually absorbed by the air, the variable $\alpha$ is also a denotation of the efficiency of the engine because it tells us, about the fraction of the energy that is absorbed by the air, out of the total energy supplied by the laser. However $\alpha$ does not give us the complete picture of the efficiency, as out of the energy absorbed by the air a fraction is used to heat the air and the rest is rejected. Let the heat rejected be $Q_{r}$. And the heat incident on or absorbed by the air is,

$$
\alpha Q=Q_{a i r}
$$

Then the efficiency of the engine becomes, 


$$
\emptyset=\left[1-\frac{Q_{r}}{\alpha Q}\right]
$$

This is the required efficiency of the engine.

\section{Experimental Setup For The Calculation Of Incident,Transmitted Energy And Bulk Temperature}

The Figure 6 below depicts the experimental setup that can be used to measure the incident energy and the transmitted energy. A one hole air injector can be set on a constant volume vessel, which will have four optical windows. The air is injected vertically downward into the vessel, A compressor can be used to compress the air. Laser induced plasma can be generated in the injected air by focussing a nano-second Q-switched Nd-YAG laser. The laser incident energy can be controlled by a half wavelength plate and a polarising beam splitter and the laser beam can be focussed by a convex lens with a suitable focal length.The incident energy and transmitted energy can be measured by energy meters, the transmitted energy can be used to estimate the energy loss caused due to attenuation and scattering. The state of plasma produced can be visualised by a ultra high speed colour camera.The injection pressure can be changed to observe the effects of the flow velocity on the properties and the diameter of the plasma. Ambient pressure can be kept constant during the experiment.Furthermore the constant volume vessel can have temperature measuring devices and pressure sensors at different sections of the vessel to calculate the temperature of the exhaust at different sections.

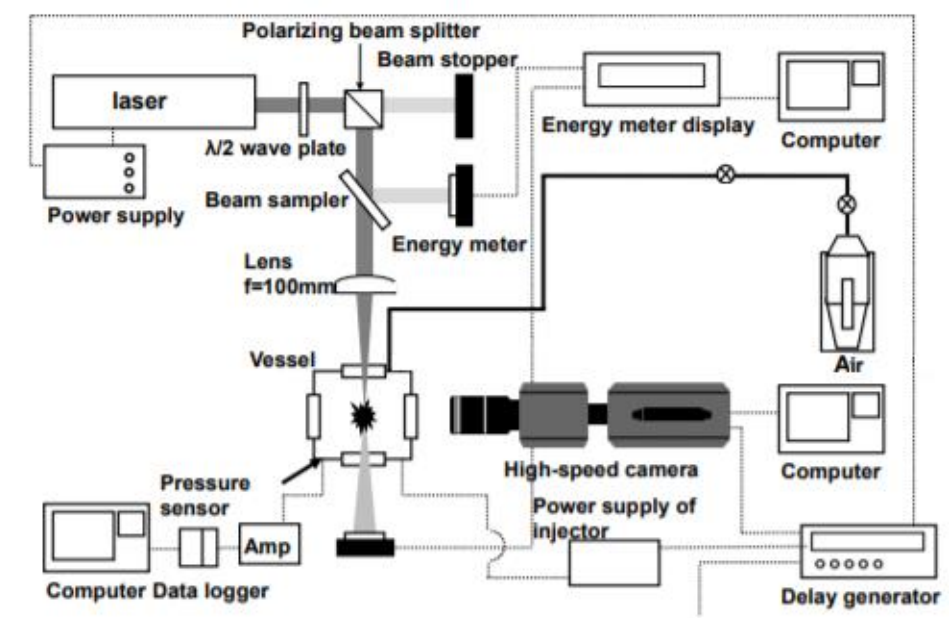

Fig. 6: Diagram edited from a similar paper [7], depicting the experimental setup in our case.

\section{Results and Disscusion}

The results obtained in the calculations are giving below. These results depict a comparative analysis between an engine based on our propulsion system and a conventional jet engine.[4]

The engine that we have used for this comparison is the "General Electric's J79".

Using the derived equations, the temperature just upstream of the turbine can be calculated for our propulsion system.Here, we assume that the nozzle and turbine contour and geometry of both the engines considered for the comparison are identical, therefore if the obtained value of temperature just upstream of the turbine is the same for both the engines, we get that the thrust produced after the expansion at the nozzle will also be the same for both the engines. Further choosing the right power rating for the laser can ensure that the air is heated to the right temperature, but in order to do so, the amount of enthalpy increase to be produced by the laser needs to be calculated. The Value of the enthalpy increase can be calculated by the equation,[12]

$$
(c \Delta T)_{a i r}=\alpha\left[\left[\Delta h+\left(\left(\frac{v_{2}^{2}}{2}-\frac{v_{1}{ }^{2}}{2}\right)\right)\right]\right]
$$


where the specific heat is at constant pressure.

Assuming the value of $\boldsymbol{\alpha}=\mathbf{1}$, and inlet velocity of air in the compressor to be $\mathbf{1 6 7 . 6 4} \mathbf{~ m} / \mathbf{s}$, as is the general value depicted by the following curve.

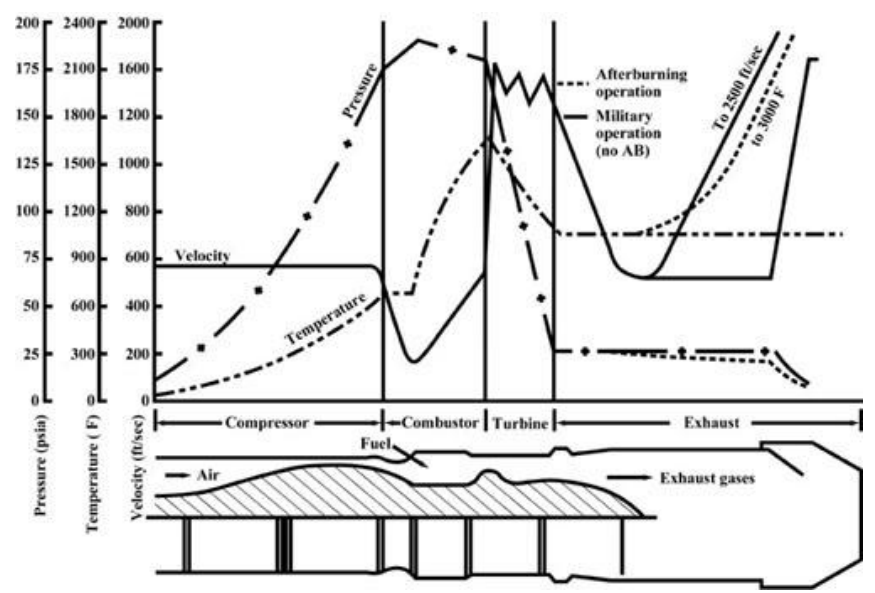

Fig. 7: Plot of engine flow parameters over the length of a turbojet[13].

From the collected data the turbine inlet temperature for the GE J79 is about, $\mathbf{8 7 1 . 1 1 1 1}^{\circ} \mathrm{C}$.

The calculated enthalpy increase to be produced by the laser used is about, $1061.94206 \mathrm{KJ} / \mathrm{Kg}$.

Hence to conclude, we can say that this propulsion system has the potential to replace the GE J79 class engines by using a laser that can impart $1061.94206 \mathrm{KJ} / \mathrm{Kg}$ increase in the enthalpy of the air.

\section{Conclusion}

A propulsion system in which the combustion chamber is replaced by a laser chamber that uses laser induced plasma as an energy source to produce thrust is proposed.

Furthermore, an experimental setup that could be used to evaluate the properties, advantages and possible shortcomings of using laser induced plasma as a source of energy was proposed.Further, a control volume analysis of the chamber is conducted and a general equation derived to calculate the temperature at different parts of the engine. Finally, the proposed propulsion system was compared to a conventional turbojet engine namely, the GE J79 and the value of enthalpy rise required for the engine to produce approximately equal thrust to the GE J79 was calculated using a basic variant derived from the standard energy equation.

\section{References}

[1] L. J. Radziemski, D. A.Cremers, Laser induced plasma: physical, chemical and biological applications. Marcel Dekker Inc., 1989.

[2] C. Phipps, M. Birkan, W. Bohn, H.-A. Eckel, H. Horisawa, T. Lippert, M. Michaelis, Y. Rezunkov, A. Sasoh, W. Schall, S. Scharring, and J. Sinko, "Review: Laser-Ablation Propulsion," Journal of Propulsion and Power, vol. 26, no. 4, pp. 609-637, 2010. DOI: 10.2514/1.43733.

[3] General Electrical J79. [Online]. Available: https://en.wikipedia.org/wiki/General_Electric_J79

[4] P. Cegelski, General Electric J79. [Online] Available: http://paulcegelski.com/j79.html

[5] Laser Induced Breakdown. [Online]. Available: https://www.rp-photonics.com/laser_induced_breakdown.html

[6] M. K. Loze, C. D. Wright, "Temperature distributions in laser-heated biological tissue with application to birthmark removal," J. of Biomedical Optics, vol. 6, no. 1, 2001. D.O.I:10.1117/1.1318217.

[7] N. Kawahara, K. Tsuboi, and E. Tomita, "Laser-induced plasma generation and evolution in a transient spray," Okayama University, Japan.

[8] D. Dundas and J. M. Rost, "Molecular Effects In Ionisation $\mathrm{OF} \mathrm{N}_{2}, \mathrm{O}_{2}$ and $\mathrm{F}_{2}$ by Intense laser fields," DOI 21.01.2005.

[9] P. K. Nag, "Engineering Thermodynamics," Tata Mc Graw Hill Education 2980 5th ed, 2013.

[10] J. H. Lienhard IV and J. H. Lienhard V, A Heat Transfer Textbook.3rd ed, 2000. 
[11] A. Narasimhan, Concept of Bulk Temperature. [Online]. Available: https://home.iitm.ac.in/arunn/docs/thermofluidsnotes-05-bulk-temp-arunn.pdf

[12] I. https://www.ohio.edu/mechanical/thermo/property_tables/air/air_Cp_Cv.html

[13] J. D. Mattingly, Elements of Propulsion: Gas Turbines and Rockets. [Online]. Available: http://soaneemrana.org/onewebmedia/ELEMENTS\%20OF\%20GAS\%20TURBINE\%20PROPULTION2.pdf 